BMJ Open Sport \& Exercise Medicine

\section{The Athletic Shoulder (ASH) test: reliability of a novel upper body isometric strength test in elite rugby players}

To cite: Ashworth B, Hogben P, Singh N, et al. The Athletic Shoulder (ASH) test: reliability of a novel upper body isometric strength test in elite rugby players. BMJ Open Sport \& Exercise Medicine 2018;4:e000365. doi:10.1136/ bmjsem-2018-000365

- Additional material is published online only. To view please visit the journal online (http://dx.doi.org/10.1136/ bmjsem-2018-000365).

Accepted 25 June 2018

\section{Check for updates}

\section{(c) Author(s) (or their} employer(s)) 2018. Re-use permitted under CC BY-NC. No commercial re-use. See rights and permissions. Published by BMJ.

${ }^{1}$ Sports Science \& Medicine Department, Arsenal Football Club, London, UK

${ }^{2}$ Sports Science \& Medicine Department, Saracens Rugby, London, UK

${ }^{3}$ Setanta College, Thurles, Ireland

${ }^{4}$ Faculty of Life Sciences, Universidad de Santander (UDES), Bucaramanga, Colombia

Correspondence to Ben Ashworth; bennyashworth@ gmail.com

\section{ABSTRACT}

Objectives Lower limb isometric tests are used to assess strength and strength asymmetries and monitor reductions in muscle force that may contribute to loss of performance and increase injury risk. Isometric tests in the upper body may be appropriate to monitor neuromuscular performance of the shoulder joint in sports involving contact and overhead actions. The aim of this study was to determine the reliability of a novel upper body isometric strength test.

Methods Eighteen elite rugby players (age 22.4 \pm 4.6 years; body mass $95.5 \pm 13.4 \mathrm{~kg}$ ) were tested on consecutive days. Maximal isometric contractions using both limbs against a force platform were assessed at three angles of abduction (180', 'l'; $135^{\circ}$, ' $Y$ ' and $90^{\circ}, \mathrm{T}^{\prime}$ '), in a prone lying position. To evaluate interday reliability, intraclass coefficients (ICC) were calculated for mean net peak force (NPF) and highest NPF achieved in any trial (peak NPF). Intratrial variability was assessed using coefficient of variation (CV), and the standard error of measurement (SEM) was used to calculate minimal detectable change (MDC).

Results Interday reliability for NPF was excellent in all test positions (ICC 0.94-0.98). The test demonstrated high absolute reliability values (SEM 4.8-10.8) and interday measurement error was below $10 \%$ in all test positions (CV 5.0-9.9\%) except for the non-dominant arm I-position (CV $11.3 \%$ ). Minimum detectable change was between 13.2 and $25.9 \mathrm{~N}$.

Conclusion The Athletic Shoulder test demonstrated excellent reliability for each test position supporting its use as a reliable tool to quantify the ability to produce and transfer force across the shoulder girdle.

\section{INTRODUCTION}

In rugby, shoulder injuries are responsible for a large number of days lost per season and have a high likelihood of recurrence. ${ }^{1}$ Injury risk is significantly increased in players during tackling and defensive drills and when required to cope with higher speeds, higher collision forces and unstable positions, as experienced in a long lever arm tackle. ${ }^{23}$ As the majority of shoulder injuries occur in the latter stages of training and matches as
What are the new findings?

- The test is a novel reliable way of assessing shoulder strength to guide safe and asymptomatic return to arm tackling as part of a return to play process.

- The test can be used to quantify recovery of muscle function after exercise or training/match exposure when compared with a player's normal values.

- It is suggested that this test forms part of a cluster of tests of shoulder function including strength, range of motion (ROM) and control, to ensure a comprehensive overview of all of the components relevant to the maintenance of shoulder stability.

a consequence of repeated exposure to tackling, fatigue may contribute to risk. ${ }^{4}$ Adequate shoulder strength may buffer against the reduced tackling ability and increased injury risk consequent to fatigue. ${ }^{5}$

In elite sports, lower limb isometric tests are used to monitor reductions in force as a potential indicator of residual or accumulated fatigue. ${ }^{6}$ Isometric tests can be routinely implemented on recovery days following a match and are potentially safer than eccentric tests with regard to minimising potential for muscle damage. ${ }^{7}$ Therefore, they can be used more regularly as a monitoring tool without concern of impacting on training and performance. In sports such as rugby or overhead sports where shoulder injuries are common, no such assessment tool exists, but such a test has potential value ${ }^{2}$ as a means to assess the athlete's capability to meet the functional demands of their sport both from an optimal performance and injury risk reduction perspective. Furthermore, objective markers are needed to inform decision making around safe return of an injured rugby player to contact and the tackle situation and to evaluate players' ability to cope with the loads they will be exposed to in competition. $^{89}$ 
Isokinetic dynamometry is a gold standard method of strength assessment but is difficult to systematically implement in sports or clinical settings ${ }^{10-13}$ resulting in the widespread use of hand-held dynamometry (HHD) to assess muscle strength. HHD is a more rapid, portable and cheaper means of evaluating shoulder strength, which can be used in a team sports environment where many players may need to be tested in a short period of time. ${ }^{14-16}$ However, the reliability of HHD is questioned due to a lack of consensus regarding test protocols and other potential sources of measurement error such as variations in warmup strategies, tester and subject position, shoulder position, tester strength, subject stabilisation and equipment stabilisation. ${ }^{17-20}$ The stabilisation of HHD during a test is affected by the relative strengths of tester and subject, and it is suggested that the reliability of the test is compromised if the subject produces more than $30 \mathrm{~kg},{ }^{21}$ an output which a substantial proportion of elite rugby players and other overhead athletes may exceed. A potential alternative to HHD is to assess isometric force using a force platform, a gold standard force-measuring tool. ${ }^{20}{ }^{22}$ Applying force into a fixed force platform would eliminate operator strength as a potential source of error. In addition, existing upper limb strength assessments are typically short lever tests that do not mimic sports specific actions or adequately assess higher shear forces experienced during competition. ${ }^{19} \mathrm{~A}$ long lever isometric test may therefore be a more appropriate means to replicate the shoulder muscle contraction required in the tackle position, ${ }^{23}{ }^{24}$ based on its correspondence to the demands of the tackle and associated mechanisms of shoulder injury. ${ }^{10}$

The primary aim of this study was therefore to establish the reliability and clinimetrics of a novel series of long lever upper body isometric force platform tests.

\section{METHODS}

Eighteen elite level male rugby players participated in the study. All participants were given a written explanation of test protocol and signed consent forms. Players were excluded if they had sustained an acute injury to the neck or shoulder girdle ( $<72$ hours prior to testing), experienced symptoms after performance of the test that lasted longer than $20 \mathrm{~min}$ or worsening pain attributed to testing or if they were unable to adopt any of the test positions due to inadequate range of motion. Body mass, height and arm length (posterior angle of the acromion to wrist joint line) were measured (online supplementary appendix 1).

\section{The IYT tests}

All tests were performed with the subject prone on the floor with their neck position standardised using a $4 \mathrm{~cm}$ foam block on which the forehead was rested. The hand was placed on a vertical axis force platform (Pasport PS-2141, Pasco Scientific, Roseville, California, USA) connected to proprietary data acquisition and analysis software (NMP technologies, London, UK). In the I-test, the shoulder was positioned in full abduction (in line with the body), the forearm in pronation and the heel of the hand acted as the main contact point with the force platform. In the Y-test and T-test, the arm was in $135^{\circ}$ and $90^{\circ}$ abduction shoulder abduction, respectively. In all tests, the elbow was fully extended (figure 1).

After a standardised warmup consisting of two submaximal 80\%-90\% efforts in each of the testing positions, ${ }^{21}$ players performed three trials in each of the three different positions on the same limb. This procedure was then repeated with the contralateral limb. During pilot testing, we established that a $20 \mathrm{~s}$ rest period was the optimal duration required for full recovery between trials, having experimented with both shorter ${ }^{17}$ and longer ${ }^{21}$ recovery times.

Subjects were required to maintain their scapula in a natural position relative to the elevated arm (avoiding excessive scapula upward rotation/elevation or anterior tilt). Subject position was checked prior to each trial to remove visible compensations. The contralateral arm was placed behind the back so that the elbow was unable to fix on the floor and provide antirotation trunk stability for the $\mathrm{Y}$ and $\mathrm{T}$ tests, but in an I-test the arm was allowed to remain by the subject's side due to lower trunk rotational forces encountered. During performance of the test, the subject was required to stabilise their trunk against rotation without using the contralateral arm and push down from the shoulder through the heel of their hand. In the instance of a failure to perform the test according to the instructions, the specific trial results were discarded and an additional trial was performed after 20 s recovery. Tests were consistently performed in the following order: I, Y, T and then conducted on consecutive days at the same time of day. Completion of all three test positions for dominant and non-dominant limbs took under 6 min including recovery periods (each individual test position for one limb took $50 \mathrm{~s}$ for example, the dominant arm T-position). No further familiarisation or upper limb training was performed between the two test days or in the 72 hours prior to the first day of testing.

In order to familiarise players with the test protocol, they performed a minimum of three complete tests on separate days prior to the study. Subjects were given standardised instructions and consistent verbal encouragement during the test, ${ }^{25}$ with a countdown prior to each of the efforts. On hearing the instruction, subjects were asked to push as fast and hard as possible to generate maximal force (aiming to achieve maximum force as fast as possible) and sustain it for the full $3 \mathrm{~s}$ test duration. ${ }^{1721}$ Tests were excluded if there was a countermovement (the hand lifted from its resting position on the platform prior to pushing down), if noticeable compensatory strategies were used, such as excessive use of the lower limb to increase stability, anterior tilt of the scapula or elbow flexion ${ }^{26}$ or if the subject did not perform the test correctly. The two examiners (Strength and conditioning 

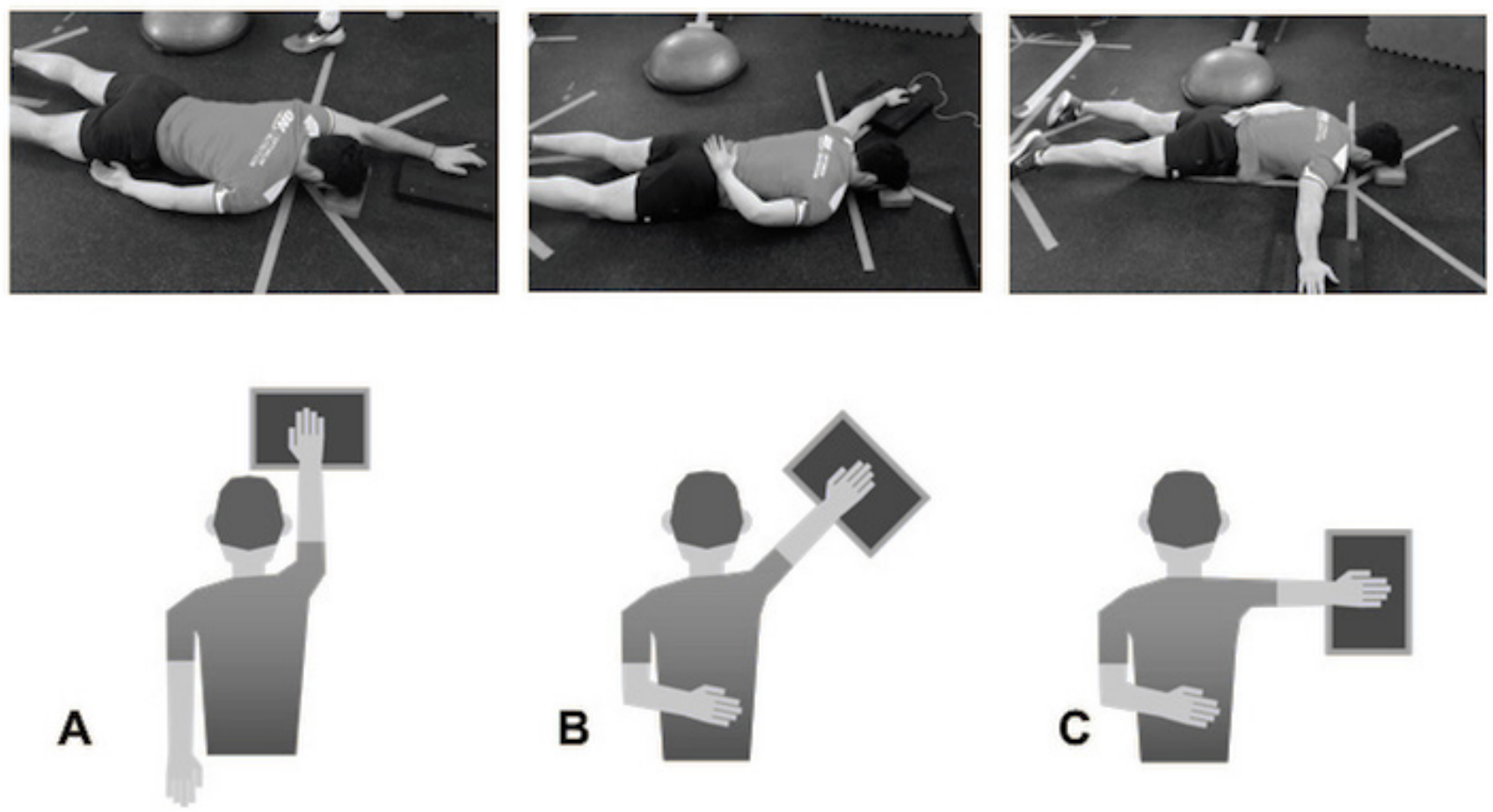

Figure 1 ASH test positions. (A) I-test (B) Y-test (C) T-test. ASH, Athletic Shoulder.

coach and Physiotherapist) were familiarised with the equipment and experienced with the test protocol.

\section{Statistical analysis}

The maximum and mean across trials of net peak force (NPF) was used in analysis. NPF=peak force achieved during the trial - force on the platform prior to the start of the contraction (i.e. weight of the limb resting on platform). Means and SD were calculated for maximum and mean NPF in Newtons (N). The data were analysed using parametric tests due to a normal distribution (Kolmogorov-Smirnov test). To assess relative reliability, the degree at which subjects maintain their position in a sample with repeated measurements; intraclass correlation coefficients (ICG) using absolute agreement were calculated. ${ }^{12}$ Values were interpreted as suggested by Fleiss, ${ }^{27}$ higher ICG values indicate better relative reliability $(>0.90=$ excellent, $0.80-0.89$ good; 0.70 and 0.79 moderate $;<0.70$ low). Absolute reliability was assessed by calculating the standard error of measurement (SEM) and minimum detectable change (MDC). ${ }^{17} 27$ The Hopkins spreadsheet for consecutive pairwise analysis of repeated measurements $^{12} 28$ was used to determine the change in mean between trials, typical error of measurement (TE) expressed as a coefficient of variation (CV\%) with $90 \%$ and $95 \%$ confidence limits. The $\mathrm{CV}$ is a reliability measure with $\leq 10 \%$ commonly used as a criterion to characterise good reliability. ${ }^{29} 30$ The MDC, the 'minimal difference needed to be considered as real', outside of the TE and corresponding to a change likely to be "almost certain" was calculated as SEM $\times 1.96 \times \sqrt{ } 2$ for $95 \%$ confidence limits or SEM $\times 1.65 \times \sqrt{ } 2$ for $90 \%$ confidence limits. ${ }^{17}$

\section{RESULTS}

Eighteen elite male rugby players participated in the study (age $22.4 \pm 4.6$ years; height $186.3 \pm 7.7 \mathrm{~cm}$; body mass $95.5 \pm 13.4 \mathrm{~kg}$ (online supplementary appendix 1-table 2). Interday reliability for mean NPF was excellent in all test positions (ICC 0.94-0.98). The test demonstrated high absolute reliability (SEM 4.8-10.8) and interday measurement error was below $10 \%$ in all test positions (CV 5.0-9.9) except for non-dominant arm I-position (CV $11.3 \%) . \mathrm{MDC}_{90}$ values for the tests are presented in table 1. Reliability was higher when we compared mean of three trials ICC $0.94-0.98$ to the maximum of three trials ICC 0.89-0.98 (online supplementary appendix 2-table 3.1 and 3.2).

\section{DISCUSSION}

We evaluated a series of novel isometric long lever shoulder tests performed on a force platform and found excellent reliability (ICCs $0.94-0.98$ ) in the three 'IYT' positions. There are no upper limb isometric force platform tests in the literature for comparison, but this compares favourably to the reliability reported for upper limb HHD tests such as internal and external rotation strength measured in a range of positions: standing, ICC: $0.92-0.96^{31}$; seated, ICC: $0.68-0.99^{32}$ and in multiple positions, ICC: $0.93-0.99 .^{17}$ Athletic Shoulder (ASH) test reliability was also higher than peak force reliability in a lower limb isometric test (ICC 0.86-0.95), ${ }^{6}$ which also used a $3 \mathrm{~s}$ maximum effort against a force platform. Based on the criteria $(\mathrm{CV}<10 \%)$ used by McCall et $a l^{6}{ }^{6}$ and others, all test positions (CV 5.0\%-9.2\%) can be 


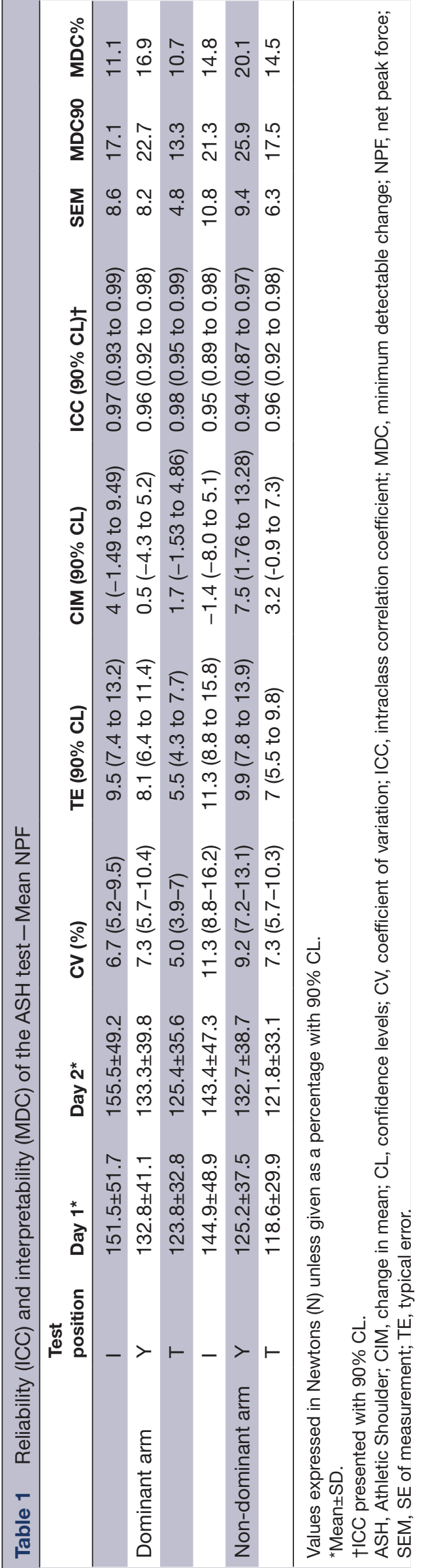

considered reliable, except for the non-dominant arm I-test (CV 11.3\%). ${ }^{30}$ Therefore, practitioners can be confident that the force platform IYT tests are reliable in detecting between subject and within subject differences.

In practical terms, high reliability does not necessarily mean that a test has the sensitivity to detect a real change, ${ }^{61017}$ and MDC is useful in a monitoring context to establish how far 'the needle needs to move' before a change can be considered meaningful. IYT values for $\mathrm{MDC}_{90}$ were 13.3-25.9 N (MDC\% ranged from 10.7 to 20.1 ), meaning that a change of $>13.3 \mathrm{~N}$ in a dominant arm T-test would be considered meaningful with a confidence level of $90 \%$. For comparison, MDC $\%$ 7.5\%-19.3\% was reported in HHD IR and ER strength tests in sitting, supine and prone positions, ${ }^{17}$ and $\mathrm{MDC} \% \leq 17.8 \%$ in prone shoulder extension. ${ }^{33}$ Holt $e t a l^{84}$ (2016) compared average internal and external rotation strength in neutral using HHD with and without external fixation (EFD) and found the range of intrarater \%MDC's were lower for HHD (17.3\%-19.7\%) than EFD (19.3\%-29.0\%). McCall et at reported $\mathrm{MDC}_{90}$ of between 26.2 and $36.9 \mathrm{n}$ across limbs and the two positions of a posterior chain isometric force platform test. Thus, the power to interpret change in the ASH test is comparable with relevant isometric tests in the literature.

In addition to reliability and interpretability, a new test should also demonstrate validity and clear purpose. ${ }^{10}$ The force platform is a gold standard force measurement tool and valid for use in assessing isometric strength. ${ }^{62}$ The principle purpose of the IYT tests was to quantify force production and potential interlimb or postinjury deficits in rugby specific shoulder positions. The T-position is similar to the 'arm tackle' position, while the I-position closely represents the 'try-scorer' injury mechanism. ${ }^{35}$ Both tests are performed using a straight arm with force delivery at the end of a long lever, corresponding to the higher torques and force transfer requirements experienced in sporting actions than the majority of short lever (bent arm) tests in the literature. Therefore, the ASH test may have value in tackling sports and in other sports, occupations and tasks that expose the shoulder to longer lever stress or require the ability to transfer forces similarly across the shoulder girdle. It is our preliminary observation from pilot testing that the long lever test demonstrated unilateral deficits that were not evident in short lever testing using HHD which warrants further investigation. To our knowledge, the only straight-arm test not using an isokinetic dynamometer but instead using HHD was developed for swimmers, with a similar subject set up to the I-test position of the current study. ${ }^{24}$ The authors reported lower interday reliability values in the dominant arm (ICC 0.81-0.95; SEM\% 4.0-8.8) than we observed in the I-position (ICC 0.95-0.97; SEM\% 5.6-7.5). Furthermore, one strength of the present study in terms of applicability to elite athletes was the assessment of reliability in an elite population, which few shoulder strength studies have done. The higher forces produced by more elite athletic populations are more 
likely to reduce HHD test reliability, ${ }^{34}$ suggesting that a stable force platform would be more appropriate for these populations, eliminating the negative influence of tester-athlete strength imbalances on reliability, particularly in stronger athletes. ${ }^{173436}$

\section{Limitations}

There were small differences in reliability between the three test positions, which could be related to different requirements of the subject to stabilise their trunk against rotational forces. Without randomisation of test order for each subject however, comparing reliability between test positions is not possible, ${ }^{19}$ and as such our study design does not allow this comparison. It could be argued that a standing test would be a more sports-specific position to evaluate the ability to produce force in a standing tackle. However, a standing test has greater potential for test-retest variability due to the additional degrees of freedom, while the prone position on the floor has the advantage of minimising the involvement of other body segments, which could explain the high reliability observed. The excellent reliability reported in the current study could also be related to the removal of inconsistencies outlined by other authors. ${ }^{19}$ In addition, trials were discarded if there were visible compensatory strategies. The duration of the test and the rest period between trials can influence results. ${ }^{26}$ In pilot testing, we determined that $20 \mathrm{~s}$ was the minimum optimal rest period needed for subjects to consistently repeat a maximum effort without deterioration in scores across the three trials. In other studies, test duration ranged from 3 to $5 \mathrm{~s}$, with rest periods ranging from $10 \mathrm{~s}^{17}$ to $5 \mathrm{~min},{ }^{33}$ factors that may have influenced intratest reliability. The authors recommend that the full ASH test protocol be completed in preseason to establish individual baselines for comparison (duration $6 \mathrm{~min}$ excluding standardised warmup). In-season monitoring can focus on one or two positions (duration 2 to $4 \mathrm{~min}$ ) to reduce time requirement. Although the method required that players were familiarised prior to testing to minimise any learning effect and 72 hours rest prior to the study protocol, it is possible that small increases in mean NPF between day 1 and day 2 were influenced either by a learning effect or additional recovery time. This emphasises the importance of familiarisation prior to interpretation of test results. It is important to recognise that results were obtained from elite male rugby players and therefore clinimetrics need to be verified in other populations.

\section{Future studies}

The portability, simplicity and rapid implementation of the test in a gym/training environment means that it has the potential to be used; with individual athletes during rehabilitation to assess progression in force production following injury; to inform return to competition decisions and to regularly monitor recovery of shoulder performance following competition induced fatigue in groups of athletes. Further work is needed to evaluate the sensitivity of the ASH test to competition induced fatigue and the profile of strength decrement and recovery as has been demonstrated in isometric lower extremity testing. ${ }^{6}$ Preliminary data in the present group of athletes suggest that the test may be a useful tool in this capacity as we noted ASH test strength deficits at 48 hours after a competitve rugby game with recovery at 72 hours. We have also observed changes beyond the $25 \mathrm{~N}$ MDC threshold in a variety of glenohumeral as well as cervical spine rehabilitation cases. However, further studies that show the sensitivity of the ASH test to alterations in training load, relationship to injury and the response to rehabilitation are the necessary next steps in determining the usefulness of this test across a wide range of environments. NPF was highest in the I-test followed by Y-test and T-test, respectively, and it may be of interest to determine normative ratios across the IYT positions in relation to sport and injury risk in larger samples.

\section{CONCLUSION}

The ASH test protocol demonstrated excellent repeatability in each test position, supporting its use as a reliable tool to quantify the ability to produce and transfer force across the shoulder girdle. Establishing individualised baseline scores and normal error (SEM and MDC) for players will permit longitudinal monitoring of change. The test may be used to benchmark performance in case of shoulder injury and to track progress during rehabilitation. It also has the potential to be used alone or in conjunction with other measures of upper limb force production to quantify athlete's readiness to perform in sports that require repeated or high intensity upper limb actions.

Acknowledgements The authors would like to thank the players of Saracens Rugby Club for their assistance and co-operation during the test period.

Contributors Conception and design: all authors. Acquisition of the data: PH, LT. Analysis and interpretation: all authors. Drafting, critical revision and final approval of the article: all authors. Statistical expertise and collection and assembly of data: BA, NS, DDC. Agreement to be accountable for all aspects of the work in ensuring that questions related to the accuracy or integrity of any part of the work are appropriately investigated and resolved: all authors.

Funding The authors have not declared a specific grant for this research from any funding agency in the public, commercial or not-for-profit sectors.

Disclaimer We wish to assure the editors that all authors included on this paper fulfil the criteria of authorship. We also provide our assurance that there is no one else who fulfils the criteria that has been excluded as an author.

Competing interests Daniel Cohen is a shareholder in NMP technologies Ltd. who have commercialised the 'ForceDecks' proprietary software used in data acquisition and analysis.

Patient consent Not required.

Ethics approval The ethics committee of the Faculty of Medicine of the University of Santander.

Provenance and peer review Not commissioned; externally peer reviewed.

Open access This is an Open Access article distributed in accordance with the Creative Commons Attribution Non Commercial (CC BY-NC 4.0) license, which permits others to distribute, remix, adapt, build upon this work non-commercially, and license their derivative works on different terms, provided the original work is properly cited and the use is non-commercial. See: http://creativecommons.org/ licenses/by-nc/4.0/ 


\section{REFERENCES}

1. Headey J, Brooks JH, Kemp SP. The epidemiology of shoulder injuries in English professional rugby union. Am J Sports Med 2007;35:1537-43.

2. Fuller $\mathrm{CW}$, Ashton $\mathrm{T}$, Brooks $\mathrm{JH}$, et al. Injury risks associated with tackling in rugby union. Br J Sports Med 2010;44:159-67.

3. Mclntosh AS, Savage TN, McCrory P, et al. Tackle characteristics and injury in a cross section of rugby union football. Med Sci Sports Exerc 2010;42:977-84.

4. Hendricks S, Matthews B, Roode B, et al. Tackler characteristics associated with tackle performance in rugby union. Eur J Sport Sci 2014;14:753-62

5. Gabbett TJ. Influence of fatigue on tackling ability in Rugby league players: role of muscular strength, endurance, and aerobic qualities. PLoS One 2016;11:e0163161.

6. McCall A, Nedelec M, Carling C, et al. Reliability and sensitivity of a simple isometric posterior lower limb muscle test in professional football players. J Sports Sci 2015;33:1298-304.

7. Lieber RL, Woodburn TM, Fridén J. Muscle damage induced by eccentric contractions of $25 \%$ strain. J Appl Physiol 1991;70:2498-507.

8. Horsley IG, Herrington LC, Rolf C. Does a SLAP lesion affect shoulder muscle recruitment as measured by EMG activity during a rugby tackle? J Orthop Surg Res 2010;5:12.

9. Usman J, Mclntosh AS, Fréchède B. An investigation of shoulder forces in active shoulder tackles in rugby union football. J Sci Med Sport 2011;14:547-52.

10. Impellizzeri FM, Marcora SM. Test validation in sport physiology: lessons learned from clinimetrics. Int J Sports Physiol Perform 2009;4:269-77.

11. Wollin M, Purdam C, Drew MK. Reliability of externally fixed dynamometry hamstring strength testing in elite youth football players. J Sci Med Sport 2016;19:93-6.

12. Hopkins WG. A scale of magnitude for effect sizes. 2002. http:// www.sportsci.org/resource/stats/effectmag.html

13. Roy JS, MacDermid JC, Orton B, et al. The concurrent validity of a hand-held versus a stationary dynamometer in testing isometric shoulder strength. J Hand Ther 2009;22:320-7.

14. McDonough A, Funk L. Can glenohumeral joint isokinetic strength and range of movement predict injury in professional rugby league. Phys Ther Sport 2014;15:91-6.

15. Rahnama N, Reilly $\mathrm{T}$, Lees $\mathrm{A}$, et al. Muscle fatigue induced by exercise simulating the work rate of competitive soccer. J Sports Sci 2003;21:933-42

16. Robineau J, Jouaux T, Lacroix M, et al. Neuromuscular fatigue induced by a $90-$ minute soccer game modeling. J Strength Cond Res 2012;26:555-62.

17. Cools AM, De Wilde L, Van Tongel A, et al. Measuring shoulder external and internal rotation strength and range of motion: comprehensive intra-rater and inter-rater reliability study of several testing protocols. J Shoulder Elbow Surg 2014;23:1454-61.

18. Hirano M, Katoh M. Absolute reliability of shoulder joint horizontal adductor muscle strength measurements using a handheld dynamometer. J Phys Ther Sci 2015;27:2125-7.
19. Schrama PP, Stenneberg MS, Lucas C, et al. Intraexaminer reliability of hand-held dynamometry in the upper extremity: a systematic review. Arch Phys Med Rehabil 2014;95:2444-69.

20. Stark T, Walker B, Phillips JK, et al. Hand-held dynamometry correlation with the gold standard isokinetic dynamometry: a systematic review. Pm $R$ 2011;3:472-9.

21. Katoh M. Test-retest reliability of isometric shoulder muscle strength measurement with a handheld dynamometer and belt. J Phys Ther Sci 2015;27:1719-22.

22. Verdera F, Champavier L, Schmidt C, et al. Reliability and validity of a new device to measure isometric strength in polyarticular exercises. J Sports Med Phys Fitness 1999;39:113-9.

23. Herrington L, Horsley I, Rolf C. Evaluation of shoulder joint position sense in both asymptomatic and rehabilitated professional rugby players and matched controls. Phys Ther Sport 2010;11:18-22.

24. Awatani T, Mori S, Shinohara J, et al. Same-session and betweenday intra-rater reliability of hand-held dynamometer measurements of isometric shoulder extensor strength. J Phys Ther Sci 2016;28:936-9.

25. Samani A, Holtermann A, Søgaard K, et al. Effects of eccentric exercise on trapezius electromyography during computer work with active and passive pauses. Clin Biomech 2009;24:619-25.

26. Maffiuletti NA, Aagaard P, Blazevich AJ, et al. Rate of force development: physiological and methodological considerations. Eur J Appl Physiol 2016;116:1091-116.

27. Kottner J, Audige L, Brorson S, et al. Guidelines for Reporting Reliability and Agreement Studies (GRRAS) were proposed. Int $J$ Nurs Stud 2011;48:661-71.

28. Hopkins WG. Spreadsheets for analysis of validity and reliability. Sportscience 2017;21.

29. Brughelli M, Van Leemputte M. Reliability of power output during eccentric sprint cycling. J Strength Cond Res 2013;27:76-82.

30. Cormack SJ, Newton RU, McGuigan MR, et al. Reliability of measures obtained during single and repeated countermovement jumps. Int J Sports Physiol Perform 2008;3:131-44.

31. Matthews MJ, Green D, Matthews H, et al. The effects of swimming fatigue on shoulder strength, range of motion, joint control, and performance in swimmers. Phys Ther Sport 2017;23:118-22.

32. Cadogan A, Laslett M, Hing W, et al. Reliability of a new hand-held dynamometer in measuring shoulder range of motion and strength. Man Ther 2011;16:97-101.

33. Awatani T, Morikita I, Shinohara J, et al. Intra- and inter-rater reliability of isometric shoulder extensor and internal rotator strength measurements performed using a hand-held dynamometer. J Phys Ther Sci 2016;28:3054-9.

34. Holt KL, Raper DP, Boettcher CE, et al. Hand-held dynamometry strength measures for internal and external rotation demonstrate superior reliability, lower minimal detectable change and higher correlation to isokinetic dynamometry than externally-fixed dynamometry of the shoulder. Phys Ther Sport 2016;21:75-81.

35. Crichton J, Jones DR, Funk L. Mechanisms of traumatic shoulder injury in elite rugby players. Br J Sports Med 2012;46:538-42.

36. Kelln BM, McKeon PO, Gontkof LM, et al. Hand-held dynamometry: reliability of lower extremity muscle testing in healthy, physically active,young adults. J Sport Rehabil 2008;17:160-70. 